\title{
Molecular contiguity between man and animal: the cutting-edge studies carried out by Paolo Sotgiu Institute, L.U.de.S. University, Lugano, Switzerland
}

\author{
Massimo Cocchi, ${ }^{1,2}$ Lucio Tonello, ${ }^{1}$ Fabio Gabrielli ${ }^{1}$ \\ ${ }^{1}$ Paolo Sotgiu Institute for Research in Quantitative \& Quantum Psychiatry \& Cardiology, L.U.de.S. \\ University, Lugano, Switzerland; ${ }^{2}$ Department of Veterinary Medical Science, University of \\ Bologna, Italy
}

\begin{abstract}
Recent research on the contiguity between man and animal provides strong evidence for the studies carried out by Paolo Sotgiu Institute for Research in Quantitative \& Quantum Psychiatry \& Cardiology, L.U.de.S. University, Lugano, Switzerland, in collaboration with the Department of Veterinary Medical Science, University of Bologna, Italy. The works, coordinated by Prof. Massimo Cocchi - Paolo Sotgiu Institute Scientific Director - have underlined this continuity, especially as to the relationship between man and dog, not only from an evolutionary point of view, but rather from a molecular perspective.
\end{abstract}

The Cambridge Declaration (7 July 2012) and its implications

The Cambridge Declaration on Consciousness concludes that The absence of a neocortex does not appear to preclude an organism from experiencing affective states. Convergent evidence indicates that nonhuman animals have the neuroanatomical, neurochemical, and neurophysiological substrates of conscious states along with the capacity to exhibit intentional behaviours. Consequently, the weight of evidence indicates that humans are not unique in possessing the neurological substrates that generate consciousness. Nonhuman animals, including all mammals and birds, and many other creatures, including octopuses, also possess these neurological substrates.

Correspondence: Massimo Cocchi, Paolo Sotgiu Institute for Research in Quantitative \& Quantum Psychiatry \& Cardiology, L.U.de.S. University, via dei Faggi 4, Lugano, Switzerland.

Tel. +41.91.985.28.30 - Fax: +41.91.994.27.05.

E-mail: massimo.cocchi@unibo.it

Key words: animal consciousness, mood disorders, Paolo Sotgiu Institute.

(C) Copyright M. Cocchi et al., 2014

Licensee PAGEPress, Italy

Journal of Biological Research 2014; 87:2126

doi:10.4081/jbr.2014.2126

This article is distributed under the terms of the Creative Commons Attribution Noncommercial License (by-nc 3.0) which permits any noncommercial use, distribution, and reproduction in any medium, provided the original author(s) and source are credited.
The above-mentioned declaration is a real turning-point in the interpretation of animal behavior since it suggests a precise relation with consciousness.

As a matter of fact, it brings about a brand new chapter for the study of animal behavior and therefore for the concept of well-being.

A critical analysis of the results obtained so far by Paolo Sotgiu Institute, L.U.de.S Unviersity and the Department of Veterinary Medical Science, University of Bologna ${ }^{1-4}$ shows evidence of molecular analogies between animal and man mood disorders. The results concerning platelet markers, together with scientific literature-based evidence, seem to substantiate the hypothesis that animals are inherently prone to mood disorders too, and, as a consequence of that, to different state of consciousness.

Supposing that consciousness is the result of a cytoskeletal quantum network processing mechanism, then it should be stated that potential consciousness can be expressed by any cell containing a cytoskeletal network, in any animal species, and this could represent the biological interface.

A hidden animal consciousness probably uses tubulin and microtubules in order to self-determine a state of consciousness limited to what is required to exist without emotional expressions and, with the development of a critical mass relationship between tubulin, synapses, cortex, and serotonin, starts leaning towards a growing neuro-correlated consciousness event (classic information) with expressions of a more complex and differentiated emotional consciousness.

It is assumed that consciousness survives even with basic conditions and this assumption is proven, at the bio-molecular level, by the hypothesis according to which Schrodinger protein (i.e. tubulin) is the biological interface from quantum physics to classic computation, the basis of quantum/classic consciousness processes and at the crossroad of memory and learning skill.

Biology and culture, consciousness and the world, subject and object, inside and outside, have continuity and find in consciousness creative transcendence and its related experiences a special level of comprehension.

Figure 1 shows the positions of animals on the self-organising map (SOM) of human depression.

The positions obtained by using the same evaluation criteria used for men ${ }^{5}$ seem to be compatible with animal features too.

\section{Remarks}

Thanks to quantum and quantitative molecular evaluations on platelet fat acids in human subjects suffering from mood disorders, and through the use of complex mathematical functions, namely the artificial neural networks (in particular the self-organizing map), it 


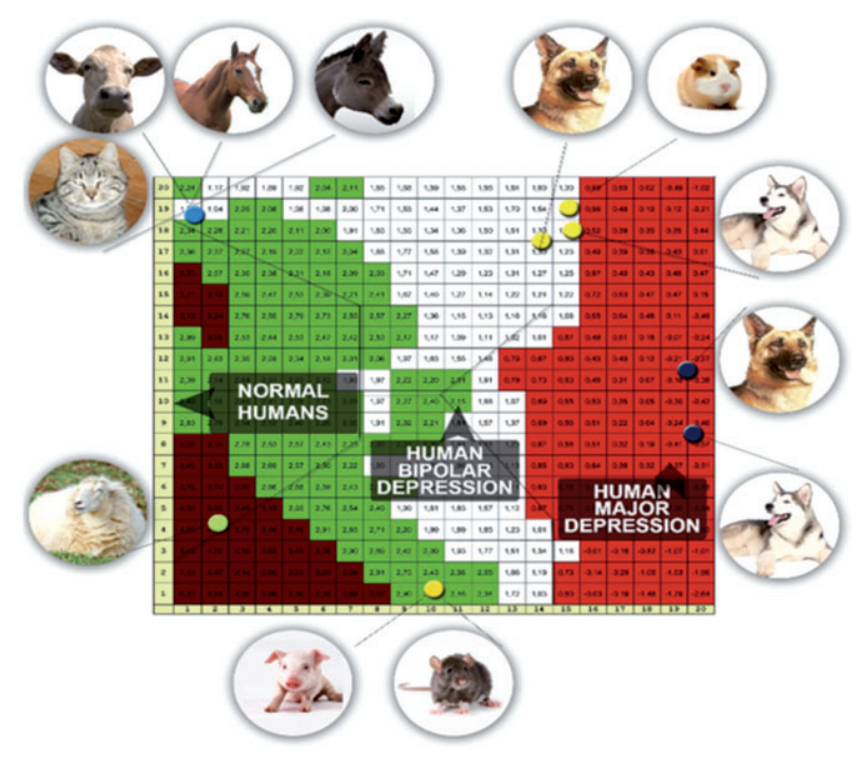

Figure 1. Positions of animals on the self-organising map of human depression. has been possible to classify subjects having unipolar depression and bipolar depression.

A similar path has been followed, in a comparable way, for some animals, and it led to interesting results for new interpretations of consciousness and animal behavior. The discovery in some dogs of the same molecular features found in human subjects suffering from major depression raises many questions about consciousness and the hypothesis stating that animals are inferior to men.

Probably, men and animals are only two different expressions of language.

The data we have, make us also believe that humans and animals (at least those we analysed) share a common biological house, which does not imply necessarily anthropocentric existential and cultural expressions.

In particular, our work shows - by going beyond animals' ability of producing forms of socialization, having feelings, behave in a sensible way - to what extent, from the biological point of view and with reference to mood disorders, animals and men share some interesting common features. In the case of major depression, the German Shepherd and the Husky have a biochemical profile similar to that of man, with all the bio-molecular implications resulting from its interpretation. And since we are convinced that there is continuity between biology and culture, the reason why the empathy between men and dogs is so strong appears crystal clear. ${ }^{6}$

\section{Conclusions}

The best way to conclude is by quoting the words on the topic of the famous Yale's psychiatrist and scientist Donald Mender: One thought that I have is to seek in wolves an olfactory (e.g. urinary pheromone), body-kinetic, or prosodic route for the expression of mood rather than a linguistic mode. The first mode might be especially fruitful, since canines seem to live in an olfactory sensory world of which we cannot even conceive, much as these creatures most likely cannot conceive of our own human linguistic universe. Can a dog write a polyphonic symphony of subtle odours? Perhaps. Might that composition feel emotionally like a Chopin etude? Why not?

\section{References}

1. Personhood Beyond the Human Conference. Available from: http://nonhumanrights.net/

2. Cocchi M, Sardi L, Tonello L, Martelli G. Do mood disorders play a role on pig welfare? Ital J Anim Sci 2009;8:691-704.

3. Cocchi M, Tonello L, Gabrielli F, et al. Quantum human and animal consciousness: a concept embracing philosophy, quantitative molecular biology \& mathematics. Journal of Consciousness Exploration \& Research 2011;2:547-74.

4. Cocchi M, Tonello L, Gabrielli F. The animal side of "mood disorders". Saarbrücken: LAP Lambert Academic Publishing; 2012.

5. Cocchi M, Tonello L. Bio molecular considerations in major depression and ischemic cardiovascular disease. Cent Nerv Sys Agents Med Chem 2010;10:97-107.

6. Berns GS, Brooks AM, Spivak M. Functional MRI in awake unrestrained dogs. PLoS ONE 2012;7:e38027. 